\title{
CURCUMIN- A BOON FOR ORAL HEALTH
}

\author{
Pramod V1, Sunil Vijay Reddy², Swati Raj33, Rishika Jaiswal4, Somudeep Tah ${ }^{5}$
}

${ }^{1}$ Reader, Department of Periodontology, New Horizon Dental College, Sakri, Bilaspur, Chhattisgarh.

2 Professor and HOD, Department of Periodontology, New Horizon Dental College, Sakri, Bilaspur, Chhattisgarh.

3 Postgraduate Student, Department of Periodontology, New Horizon Dental College, Sakri, Bilaspur, Chhattisgarh.

${ }_{4}^{4}$ Postgraduate Student, Department of Periodontology, New Horizon Dental College, Sakri, Bilaspur, Chhattisgarh.

5 Postgraduate Student, Department of Periodontology, New Horizon Dental College, Sakri, Bilaspur, Chhattisgarh.

ABSTRACT
BACKGROUND
Turmeric is a spice derived from the rhizomes of Curcuma longa, which is a member of the ginger family (Zingiberaceae). In
addition to its use as a spice and pigment, Turmeric has been used for centuries in herbal medicines for the treatment of a variety
of disorders such as rheumatism, diabetic ulcers, anorexia, cough and sinusitis. Curcumin (Diferuloylmethane) is the main active
constituent present in turmeric, responsible for its yellow colour. It has been shown to possess significant anti-inflammatory, anti-
oxidant, anti-carcinogenic, anti-mutagenic, anticoagulant and anti-infective effects.

\section{KEYWORDS}

Curcumin, Anti-Inflammatory, Antimicrobial, Anticarcinogenic.

HOW TO CITE THIS ARTICLE: Pramod V, Reddy SV, Raj S, et al. Curcumin- a boon for oral health. J. Evolution Med. Dent. Sci. 2018;7(13):1668-1672, DOI: 10.14260/jemds/2018/376

\section{BACKGROUND}

From ancient times, plants have been an excellent source of therapeutic as well as chemotherapeutic agents. ${ }^{1}$ Curcumin [1, 7-bis-(4-hydroxy-3-methoxyphenyl)-1, 6-heptadiene-3, 5dione] is one of a natural polyphenolic yellow coloured compound isolated from the rhizome of Curcuma longa Linn (turmeric) (family Zingiberaceae), which has been used for centuries as a dietary pigment, spice and traditional medicine in India and China.2,3,4 Curcuma longa rhizome has been traditionally used as antimicrobial agent as well as an insect repellent. ${ }^{5}$ Rhizomes are horizontal underground stems that send out shoots as well as roots. The bright yellow colour of turmeric comes mainly from fat-soluble, polyphenolic pigments known as curcuminoids. ${ }^{6}$ Diferuloylmethane known as curcuminoid is major constituent of curcumin (77 wt\%); the other two curcuminoids are demethoxycurcumin (17 wt\%) and bisdemethoxycurcumin (3wt\%). ${ }^{7}$ It is a common spice, known mostly for its use in Indian dishes as a common ingredient in curries and other ethnic meals. Turmeric has also been used for centuries in Ayurvedic medicine, which integrates the medicinal properties of herbs with food. This extraordinary herb has found its way into the spotlight in the west, because of its wide range of medicinal benefits. ${ }^{6}$

'Financial or Other Competing Interest': None.

Submission 06-02-2018, Peer Review 10-03-2018,

Acceptance 17-03-2018, Published 26-03-2018.

Corresponding Author:

Dr. Pramod V,

Reader, Department of Periodontology,

New Horizon Dental College,

Sakri, Bilaspur,

Chhattisgarh.

E-mail: pramdm@gmail.com

DOI: $10.14260 /$ jemds $/ 2018 / 376$

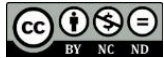

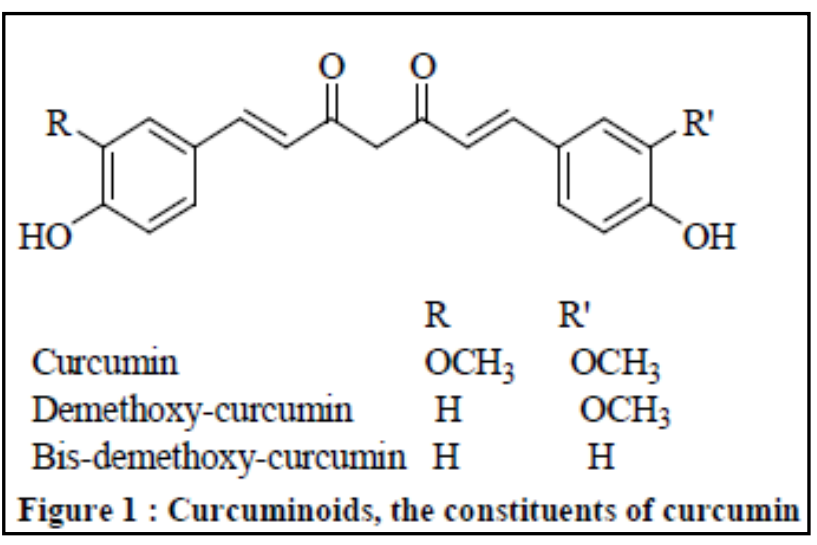

Curcumin is a hydrophobic polyphenol with two carbonyl groups and several studies have revealed extremely low water-solubility, low stability, rapid metabolism and poor absorption of this molecule due to the presence of two carbonyl atoms that severely reduces its bioavailability and consequently decreasing the health benefits related to this vital compound. ${ }^{8-10}$ Hence, to overcome this problem several monocarbonyl derivatives and drug delivery systems have also been discovered.11-12 It is considered to be a safe phytochemical owing non-toxic, non-genotoxic and nonteratogenic properties even at high doses. ${ }^{13}$

Curcumin can exist in at least two tautomeric forms, keto and enol. The enol form is more energetically stable in the solid phase and in solution. Curcumin is brightly yellow coloured and may be used as a food colouring. As a food additive, its E number is E100.6

\section{Properties of Curcumin}

Curcumin and its derivatives owns a broad spectrum of therapeutic activities viz. antibacterial, antifungal, antiviral, anti-HIV, anti-inflammatory, anti-Parkinson's, antiAlzheimer's, anti-angiogenesis, free radical scavenging activity, antirheumatic, antimalarial, anticancer, antiprotozoan, antimutagenic, wound treatment, hepatoprotective activity, anti-leishmanial activity and amyloid heart disease. Recent reports have revealed that 
curcumin helps in decreasing total cholesterol and LDL cholesterol level in serum and increases the beneficial HDL cholesterol level. ${ }^{7}$

Curcumin exerts anti-inflammatory activity by inhibition of a number of different molecules that play an important role in inflammation. Turmeric is effective in reducing postsurgical inflammation. Turmeric helps to prevent atherosclerosis by reducing the formation of blood clumps.

Curcumin inhibits the growth of Helicobacter pylori, which causes gastric ulcers and has been linked with gastric cancers. Curcumin can bind with heavy metals such as cadmium and lead, thereby reducing the toxicity of these heavy metals. This property of Curcumin explains its protective action to the brain. Curcumin acts as an inhibitor for cyclooxygenase, 5-lipoxygenase and glutathione Stransferase.

Turmeric is a potent antioxidant. Curcumin, its main active constituent is as powerful and antioxidant as vitamins $\mathrm{C}, \mathrm{E}$ and Beta-Carotene, making turmeric usage a consumer choice for cancer prevention, liver protection and premature aging. In addition, turmeric is a powerful anti-inflammatory, easing conditions such as bursitis, arthritis and back pain. Turmeric's anti-inflammatory action is likely due to a combination of three different properties. First, turmeric lowers the production of inflammation-inducing histamine. Secondly, it increases and prolongs the action of the body's natural anti-inflammatory adrenal hormone, cortisol and finally, turmeric improves circulation, thereby flushing toxins out of small joints where cellular wastes and inflammatory compounds are frequently trapped. Research has also confirmed the digestive benefits of turmeric. Turmeric acts as a cholagogue, stimulating bile production thus increasing the body's ability to digest fats, improving digestion and eliminating toxins from the liver. ${ }^{6}$

\section{Active Constituents}

The active constituents of turmeric are the flavonoid curcumin (Diferuloylmethane) and various volatile oils including tumerone, atlantone and zingiberene. Other constituents include sugars, proteins and resins. The bestresearched active constituent is curcumin, which comprises 0.3 - 5.4 percent of raw turmeric. 6

\section{Pharmacokinetics}

Pharmacokinetic studies in animals have demonstrated that 40 - 85 percent of an oral dose of curcumin passes through the gastrointestinal tract unchanged with most of the absorbed flavonoid being metabolised in the intestinal mucosa and liver. Due to its low rate of absorption, curcumin is often formulated with bromelain for increased absorption and enhanced anti-inflammatory effect. 6

\section{Mechanisms of Action/ Antioxidant Effects}

Water- and fat-soluble extracts of turmeric and its curcumin component exhibit strong antioxidant activity comparable to vitamins $C$ and $E$. A study of ischaemia in the feline heart demonstrated that Curcumin pre-treatment decreased ischaemia-induced changes in the heart. An in vitro study measuring the effect of curcumin on endothelial hemeoxygenase- 1 , an inducible stress protein was conducted utilising bovine aortic endothelial cells. Incubation (18 hours) with curcumin resulted in enhanced cellular resistance to oxidative damage.

\section{Hepatoprotective Effects}

Turmeric has been found to have a hepatoprotective characteristic similar to silymarin. Animal studies have demonstrated turmeric's hepatoprotective effects from a variety of hepatotoxic insults including carbon tetrachloride (CCl4), galactosamine, acetaminophen (Paracetamol) and Aspergillus aflatoxin. Turmeric's hepatoprotective effect is mainly a result of its antioxidant properties as well as its ability to decrease the formation of pro-inflammatory cytokines. In rats with CCl4-induced acute and subacute liver injury, curcumin administration significantly decreased liver injury in test animals compared to controls. Turmeric extract inhibited fungal aflatoxin production by 90 percent when given to ducklings infected with Aspergillus parasiticus. Turmeric and curcumin also reversed biliary hyperplasia, fatty changes and necrosis induced by aflatoxin production. Sodium curcuminate, a salt of curcumin, also exerts choleretic effects by increasing biliary excretion of bile salts, cholesterol and bilirubin as well as increasing bile solubility, therefore possibly preventing and treating cholelithiasis.

\section{Anti-inflammatory Effects}

The volatile oils and curcumin of Curcuma longa exhibit potent anti-inflammatory effects. Oral administration of curcumin in instances of acute inflammation was found to be as effective as cortisone or phenylbutazone, and one-half as effective in cases of chronic inflammation. In rats with Freund's adjuvant-induced arthritis, oral administration of Curcuma longa significantly reduced inflammatory swelling compared to controls. In monkeys, curcumin inhibited neutrophil aggregation associated with inflammation. C. longa's anti-inflammatory properties may be attributed to its ability to inhibit both biosynthesis of inflammatory prostaglandins from arachidonic acid and neutrophil function during inflammatory states. Curcumin may also be applied topically to counteract inflammation and irritation associated with inflammatory skin conditions and allergies, although care must be used to prevent staining of clothing from the yellow pigment.

\section{Anticarcinogenic Effects}

Animal studies involving rats and mice as well as in vitro studies utilising human cell lines have demonstrated curcumin's ability to inhibit carcinogenesis at three stages: tumour promotion, angiogenesis and tumour growth. In two studies of colon and prostate cancer, curcumin inhibited cell proliferation and tumour growth. Turmeric and curcumin are also capable of suppressing the activity of several common mutagens and carcinogens in a variety of cell types in both in vitro and in vivo studies. The anticarcinogenic effects of turmeric and curcumin are due to direct antioxidant and freeradical scavenging effects as well as their ability to indirectly increase glutathione levels, thereby aiding in hepatic detoxification of mutagens and carcinogens and inhibiting nitrosamine formation.

\section{Antimicrobial Effects}

Turmeric extract and the essential oil of Curcuma longa inhibit the growth of a variety of bacteria, parasites and 
pathogenic fungi. A study of chicks infected with the caecal parasite Eimeria maxima demonstrated that diets supplemented with 1-percent turmeric resulted in a reduction in small intestinal lesion scores and improved weight gain. Another animal study, in which guinea pigs were infected with either dermatophytes, pathogenic molds or yeast found that topically applied turmeric oil inhibited dermatophytes and pathogenic fungi, but neither curcumin nor turmeric oil affected the yeast isolates. Improvements in lesions were observed in the dermatophyte- and fungiinfected guinea pigs, and at seven days post-turmeric application the lesions disappeared. Curcumin has also been found to have moderate activity against Plasmodium falciparum and Leishmania major organisms.

\section{Cardiovascular Effects}

Turmeric's protective effects on the cardiovascular system include lowering cholesterol and triglyceride levels, decreasing susceptibility of low density lipoprotein (LDL) to lipid peroxidation and inhibiting platelet aggregation. These effects have been noted even with low doses of turmeric. A study of 18 atherosclerotic rabbits given low-dose (1.6 - 3.2 $\mathrm{mg} / \mathrm{kg}$ body weight daily) turmeric extract demonstrated decreased susceptibility of LDL to lipid peroxidation, in addition to lower plasma cholesterol and triglyceride levels. The higher dose did not decrease lipid peroxidation of LDL, but cholesterol and triglyceride level decreases were noted, although to a lesser degree than with the lower dose. Turmeric extract's effect on cholesterol levels may be due to decreased cholesterol uptake in the intestines and increased conversion of cholesterol to bile acids in the liver. Inhibition of platelet aggregation by $\mathrm{C}$. longa constituents is thought to be via potentiation of prostacyclin synthesis and inhibition of thromboxane synthesis.

\section{Gastrointestinal Effects}

Constituents of Curcuma longa exert several protective effects on the gastrointestinal tract. Sodium curcuminate inhibited intestinal spasm and p-tolymethylcarbinol, a turmeric component, increased gastrin, secretin, bicarbonate and pancreatic enzyme secretion. Turmeric has also been shown to inhibit ulcer formation caused by stress, alcohol, indomethacin, pyloric ligation and reserpine, significantly increasing gastric wall mucus in rats subjected to these gastrointestinal insults. Curcumin enhances immunity. Curcumin can also help the body fight off cancer should some cells escape apoptosis. When researchers looked at the lining of the intestine after ingestion of curcumin, they found that CD4+ T-helper and B type immune cells were greater in number. In addition to this localised immune stimulation, curcumin also enhances immunity in general. Researchers in India have documented increased antibodies and more immune action in mice when given curcumin.

\section{Curcumin blocks NF- $\mathrm{B}$ and the Motogenic Response in} Helicobacter Pylori-Infected Epithelial Cells

Studies indicate that infection of epithelial cells by the microbial pathogen Helicobacter pylori leads to activation of the transcription factor nuclear factor $\kappa B(N F-\kappa B)$, the induction of pro-inflammatory cytokine/ chemokine genes and the motogenic response (Cell Scattering). It has been investigated that $\mathrm{H}$. pylori-induced NF- $\mathrm{KB}$ activation and the subsequent release of interleukin 8 (IL-8) are inhibited by curcumin (Diferuloylmethane), a yellow pigment in turmeric (Curcuma Longa L). It has been demonstrated that curcumin inhibits I $\mathrm{K} \alpha$ degradation, the activity of IкB kinases $\alpha$ and $\beta$ (IKK $\alpha$ and $\beta$ ), and NF-кB DNA-binding. The mitogen-activated protein kinases (MAPK), extracellular signal-regulated kinases 1/2 (ERK1/2) and p38, which are also activated by $\mathrm{H}$. pylori infection are not inhibited by curcumin. It is studied that $\mathrm{H}$. pylori-induced motogenic response is blocked by curcumin. It has been concluded that curcumin due to inhibition of NF- $\kappa \mathrm{B}$ activation and cell scattering should be considered as a potential therapeutic agent effective against pathogenic processes initiated by $\mathrm{H}$. pylori infection.

\section{Pregnancy and Lactation}

Although, there is no evidence that dietary consumption of turmeric as a spice adversely affects pregnancy or lactation, the safety of curcumin supplements in pregnancy and lactation has not been established. ${ }^{6}$

\section{Enhancing the Bioavailability and Solubility of Curcumin to Improve Antimicrobial Activities}

The optimum potential of curcumin is limited because of poor oral bioavailability and insufficient solubility in aqueous solvents leading to poor absorption, fast metabolism and quick systemic elimination. ${ }^{14,15}$ For overcoming this obstacle, nanocarriers like curcumin-loaded PLGA (poly lactide-coglycolide) and curcumin nanoparticle formulation were investigated and their better bioactivity and bioavailability as well as increased cellular uptake compared to Curcumin were reported. Another study revealed that heat-extracted curcumin elevated the solubility of curcumin 12-folds without significant disintegration due to heat treatment. Modification of 4-hydroxy-2-nonenal (HNE) as a critical oxidation byproduct involved in disease pathogenesis via cytotoxicity, genotoxicity and mutagenicity is inhibited $80 \%$ by heat solubilised Curcumin and suggested a possible mechanism for inducing bioactivity of curcumin. ${ }^{16}$ The study of nanocurcumin as a nanoparticle of curcumin with the size of $2.40 \mathrm{~nm}$ processed by a wet-milling technique showed curcumin to be more freely dispersible in water leading to more significant antimicrobial activity against Staph. aureus, E. coli, P. aeruginosa, B. subtilis and two fungi of P. notatum and $A$. niger due to reduced particle size and enhanced bioavailability. ${ }^{17,18}$ However, nanocurcumin demonstrated more noteworthy activity against Gram-positive bacteria rather than Gram negatives. ${ }^{17}$ In another study to improve the stability and solubility of curcumin, microencapsulation process was investigated. Microcapsule of curcumin with improved solubility is suitable as a preservative and colorant in food industry and it exhibited potent antimicrobial effect against food-borne pathogens including E. coli, Staph. aureus, B. subtilis, B. cereus, Yersinia enterocolitica, Penicillium notatum and Saccharomyces cerevisiae with MIC values ranging from 15.7 to $250 \mathrm{~g} / \mathrm{mL}$. It was demonstrated that Gram-positive bacteria were more susceptible to the microcapsulated Curcumin compared to Gram-negatives. However, antifungal effect was found to be stronger than the bactericidal effect. ${ }^{19,20}$ 


\section{Adverse Events Associated with Curcumin}

Although, curcumin has been shown to exhibit beneficial activities in a plethora of human diseases with minimal toxicities, some investigators have reported undesired adverse effects associated with this polyphenol. Lao et al 21 conducted a dose-escalation study to determine the maximum tolerable dose and safety of a single oral dose of curcumin in 34 healthy volunteers. The volunteers were given escalating doses of curcumin ranging from 500 to $12,000 \mathrm{mg}$, and safety was assessed for $72 \mathrm{~h}$ after administration. Twenty-four participants completed the trial, seven of whom experienced minimal toxicity that did not appear to be dose-related. More specifically, these seven participants experienced diarrhoea, headache, rash and yellow stool.22

In another study, curcumin at doses ranging from 0.45 to $3.6 \mathrm{~g} /$ day for 1 to 4 months was associated with nausea and diarrhoea and caused an increase in serum alkaline phosphatase and lactate dehydrogenase contents in human participants. ${ }^{23}$ In patients with high-risk or premalignant lesions, doses of curcumin above $8 \mathrm{~g} /$ day were unacceptable to patients because of the bulky volume of the tablets. ${ }^{24}$ In one study of patients with advanced pancreatic cancer, 5 of the 17 patients receiving curcumin $(8 \mathrm{~g} /$ day) in combination with gemcitabine reported intractable abdominal pain after a few days to 2 weeks of curcumin intake. 25 Thus, more studies are required to evaluate the long-term toxicity associated with curcumin before it can be approved for human use.

\section{CONCLUSION}

As discussed in this review, curcumin has shown therapeutic potential against a number of human diseases. The underlying mechanism for curcumin's clinical efficacy seems to be modulation of numerous signaling molecules. However, because of the complex nature of the diseases, the underlying mechanism in many cases remains unclear. Poor bioavailability and limited adverse effects reported by some investigators are a major limitation to the therapeutic utility of curcumin. We hope that the results from ongoing clinical trials will provide a deeper understanding of curcumin's therapeutic potential and will help to place this fascinating molecule at the forefront of novel therapeutics.

\section{REFERENCES}

[1] Singh A, Kaur N, Sharma S, et al. Recent progress in biologically active xanthones. J Chem Pharm Res 2016;8(1):75-131.

[2] Kuttan R, Bhanumathy P, Nirmala $K$, et al. Potential anticancer activity of turmeric (Curcuma longa). Cancer Lett 1985;29(2):197-202.

[3] Kuttan R, Sudheeran PC, Josph CD. Turmeric and curcumin as topical agents in cancer therapy. Tumori 1987;73(1):29-31.

[4] Li Y, Zhang T. Targeting cancer stem cells by curcumin and clinical applications. Cancer Lett 2014;346(2):197-205.

[5] Moghadamtousi SZ, Kadir HA, Hassandarvish P, et al. A review on antibacterial, antiviral and antifungal activity of curcumin. Biomed Res Int 2014;2014:1-12.

[6] Akram M, Shahab-Uddin, Ahmed A, et al. Curcuma longa and curcumin: a review article. Rom J Biol-Plant Biol 2010;55(2):65-70.
[7] Kaur N, Kumar M, Dhiman S, et al. Curcumin and its derivatives as chemotherapeutic agents. J Chem Pharm Res 2016;8(2):301-18.

[8] Yang C, Su X, Liu A, et al. Advances in clinical study of curcumin. Curr Pharm Des 2013;19(11):1966-73.

[9] Aggarwal BB, Sung B. Pharmacological basis for the role of curcumin in chronic diseases: an age-old spice with modern targets. Trends Pharmacol Sci 2009;30(2):85-94.

[10] Anand P, Kunnumakkara AB, Newman RA, et al. Bioavailability of curcumin: problems and promises. Mol Pharm 2007;4(6):807-18.

[11] Bisht S, Maitra A. Systemic delivery of curcumin: 21st century solutions for an ancient conundrum. Curr Drug Discov Technol 2009;6(3):192-9.

[12] Gou M, Men K, Shi $H$, et al. Curcumin-loaded biodegradable polymeric micelles for colon cancer therapy in vitro and in vivo. Nanoscale 2011;3(4):1558-67.

[13] Soni KB, Kuttan R. Effect of oral curcumin administration on serum peroxides and cholesterol levels in human volunteers. Indian J Physiol Pharmacol 1992;36(4):273-5.

[14] Mohanty C, Das M, Sahoo SK. Emerging role of nanocarriers to increase the solubility and bioavailability of curcumin. Expert Opinion on Drug Delivery 2012;9(11):1347-64.

[15] Anand P, Nair HB, Sung B, et al. Design of curcuminloaded PLGA nanoparticles formulation with enhanced cellular uptake, and increased bioactivity in vitro and superior bioavailability in vivo. Biochemical Pharmacology 2010;79(3):330-8.

[16] Kurien BT, Singh A, Matsumoto H, et al. Improving the solubility and pharmacological efficacy of curcumin by heat treatment. Assay Drug Dev Technol 2007;5(4):567-76.

[17] Bhawana, Basniwal RK, Buttar HS, et al. Curcumin nanoparticles: preparation, characterization and antimicrobial study. J Agric Food Chem 2011;59(5):2056-61.

[18] Shailendiran D, Pawar N, Chanchal A, et al. Characterization and antimicrobial activity of nanocurcumin and curcumin. Proceedings of the International Conference on Nanoscience, Technology and Societal Implications (NSTSI '11) 2011: p. 1-7.

[19] Wang Y, Lu Z, Wu H, et al. Study on the antibiotic activity of microcapsule curcumin against foodborne pathogens. Int J Food Microbiol 2009;136(1):71-4.

[20] Wang YF, Shao JJ, Zhou CH, et al. Food preservation effects of curcumin microcapsules. Food Control 2012;27(1):113-17.

[21] Lao CD, Ruffin MT, Normolle D, et al. Dose escalation of a curcuminoid formulation. BMC Complement Altern Med 2006;6:10.

[22] Gupta SC, Patchva S, Aggarwal BB. Therapeutic roles of curcumin: lessons learned from clinical trials. The American Association of Pharmaceutical Scientists Journal 2013;15(1):195-218.

[23] Sharma RA, Euden SA, Platton SL, et al. Phase I clinical trial of oral curcumin: biomarkers of systemic activity and compliance. Clin Cancer Res 2004;10(20):684754. 


\section{Jemds.com}

[24] Cheng AL, Hsu CH, Lin JK, et al. Phase I clinical trial of curcumin, a chemopreventive agent, in patients with high-risk or pre-malignant lesions. Anticancer Res 2001;21(4B):2895-900.

\section{Review Article}

[25] Epelbaum R, Schaffer M, Vizel B, et al. Curcumin and gemcitabine in patients with advanced pancreatic cancer. Nutr Cancer 2010;62(8):1137-41. 